\title{
La similitude des transports de sédiments sous l'action simultanée de la houle et des courants
}

\author{
Similitude laws for sediment transport \\ under the simultaneous action of waves and currents
}

\author{
PAR J. GODDET ET P. JAFFRY \\ INGËNIEURS AU LABORATOIRE NATIONAL D'HYDRAULIQUE DE CHATOU
}

\begin{abstract}
Action des courants permanents: rappel des règles de similitude et de leurs applications. Action de la houle: état de la question; études du Laborutoire National d'Hydraulique permettant de déterminer, dans certaines conditions, les vitesses critiques de début d'entruinement. Action simultanée de la houle et des courants : étude de la compatibilité des conditions de similitude respectives en vue de la prédétermination des caractéristiques des modèles réduits. Vérification expérimentale.
\end{abstract}

\begin{abstract}
The action of permanent currents: a review of similitude laws and their applications.

Wave action: present state of the problem; studies performed at the Laboratoire National d'Hydraulique to enable determination of transport threshold speeds under certain conditions. The simultaneous action of waves and currents: investigation of the compatibility of the respective similitude conditions to enable the predetermination of scale model characteristirs. Experimental check.
\end{abstract}

Communication présentée à la Société Hydrotechnique de France le 18 juin 1959

Les mouvements de sédiments dans un domaine maritime déterminé font souvent intervenir simultanément l'action de la houle et des courants permanents ou lentement variables, que ceux-ci soient dus à la proximité d'un fleuve côtier, à la marée ou à toute autre cause.

Lorsqu'un tel problème est soumis à une étude expérimentale sur modèle réduit, on représente généralement les sédiments par un matériau mobile dont les caractéristiques sont définies par fâtonmements, de manière à reprodruire aussi fidèlement que possible les phénomènes naturels. Cette phase de l'étude constitue l'étalonnage du modèle qui est purement empirique.

Différents modèles, maritimes à fonds mobiles, mettant en jeu la houle et les courants, ont été réalisés au Laboratoire National d'Hydraulique. Par ailleurs, l'étude générale du transport des sédiments par les courants, puis par la houle, a été poursuivie tant expérimentalement que sur le plan théorique.
En ce qui concerne le charriage sous l'action des courants, les travaux d'un certain nombre dc chercheurs mettent à notre disposition plusieurs formules qui, si elles ne sont pas rigoureusement identiques, sont cependant assez bien vérifiées par l'expérience, tant en nature que sur modèle, pour permettre de définir les conditions de similitude de charriage.

En ce qui concerne le transport par la houle, les études effectuées au Laboratoire National d'Hydraulique nous fournissent des notions relativement précises, tant sur la vitesse de début d'entraînement que sur le débit solide, tout au moins à l'échelle des houles de laboratoire. Pour autant que ces lois puissent être étendues aux houles naturelles, elles permettent également de définir une similitude de charriage par la houle.

Le but de la présente note est d'examiner dans quelles conditions les lois de similitude du charriage, respectivement sous l'action des courants et de la houle, sont compatibles, selon 
que l'on envisage un modèle réduit sans distorsion ou avec distorsion et selon qu'il s'agit de matéliaux fins, comme les sables, ou nettement plus gros, comme les galets.

Dans l'examen de ces différents cas, nous nous référerons aux études sur modèle effectuées à Chatou, qui, rappelons-le, sont antérieures à la présente mise au point.

Il s'agit donc davanlage d'un essai de systématisation, qui reste à soumettre à l'épreuve de l'expérience, et qui ne prétend pas résoudre définitivement le problème de la similitude du charriage, ne serait-ce qu'à cause de l'étroitesse du domaine dans lequel les lois proposées ont été établies.

Nous examinerons successivement :

Les lois relatives au charriage par les cou- rants, établies par différents auteurs (MeyerPeter, Shields, Eguiasaroff) et leurs conséquences sur la similitude;

-- Les lois relatives au charriage par la houle, étudiées à Chatou ou au cours de recherches antérieures, et la possibilité de les représenter en similitude;

-. Enfin, les conditions de compatibilité de ces deux similitudes, dans les deux cas suivants :

a) Pour des sédiments de dimensions relativement grandes vis-à-vis de l'ćpaisseur de la couche-limite oscillatoire : galets;

b) Pour des sédiments de dimensions relativement petites vis-à-vis de l'épaisseur de la couche-limite oscillatoire : sables.

\section{1. - LOIS DE CHARRIAGE SOUS L'ACTION DES COURANTS PERMANENTS}

Nous utiliserons les notations suivantes :

ॠ : poids spécifique de l'eau;

$\sigma^{\prime}{ }_{g}$ : poids spécifique des matériaux de fond pesés sous l'eau;

D : diamètre moyen du matériau;

$h$ : tirant d'eau;

J : pente de la ligne d'eau;

$\tau \quad$ : force tractrice;

$\tau_{0}$ : force tractrice critique, correspondant au début de charriage;

$g_{s}^{\prime} \quad$ : débit solide en poids, par mètre de largeur, pesé sous l'eau;

A : coefficient numérique compris entre 0,03 et 0,05 ;

$q$ : débit liquide en poids par mètre de largeur;

et les échelles de similitude suivantes :

$\lambda \quad$ : longueurs horizontales;

$\mu \quad$ : hauteurs;

$\zeta=\mu / \dot{\lambda} \quad:$ distorsion;

$0_{n}=\mu^{1 / 2} \xi^{-1}$ : temps hydrauliques;

$\theta \quad:$ temps de charriage;

$\eta \quad$ : masse en poids spécifiques apparents du matériau sous l'eau;

§ : diamètre du matériau.

\section{A) Formule de Meyer-Peter :}

C'est une formule expérimentale établie pour un écoulement permanent. Elle s'écrit :

$$
0,25(\sigma / g)^{1 / 3} g_{s}^{2 / 3}=\tau \cdots \tau_{11}
$$

arec : $\tau=\bar{\omega} \mathrm{h} \mathrm{J}$

$\tau_{0}=A \sigma_{s}^{\prime} D$ où $A$ dépend du nombre de Reynolds du grain $R=u_{*} \mathrm{D} / \mathrm{v}$ avec $u_{*}=\sqrt{\tau_{n} / \rho}$, A restant voisin de 0,05 pour $\mathcal{R} \geqslant 900$.

La formule est applicable avec des valeurs légèrement inférieures de $A$ pourvu que $\mathfrak{R} \geqslant 60$.

Il en découle les conséfuences suivantes :

1) Au début d'entraînement, $\tau=\tau_{0}$;

2) Pour le charriage établi, quand $\tau>\tau_{\theta}$, la formule permet de calculer le débit solide $g_{s}^{\prime}$;

3) Les conditions de similitude suivantes, qui résultent de l'application de la formule (1) au prototype et au modèle :

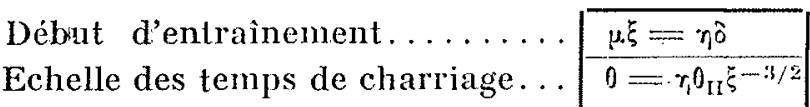

\section{B) Formule d'Eguiasaroff :}

C'est une formule théorique, en bon accord avec les résultats obtenus par Shields en ce qui concerne la vitesse de début d'entraînement.

Elle s'écrit :

$$
g_{s}^{\prime}=0,015 \bar{\omega} q \mathrm{~J}^{1 / 2} \mathrm{~K} \frac{\tau-\tau_{0}}{\tau_{f ;}}
$$


avec :

$\tau_{0}=A \bar{\omega}_{s}^{\prime} \mathrm{D}$, et $k$ un coefficient égal à 1 pour $\mathcal{R} \geqslant 60$.

Conséquences :

1) Début d'entraînement : $\tau=\tau_{0}$.

2) Charriage établi si $\tau>\tau_{0}$ la formule donne $g^{\prime}$.

3) Conditions de similitude :

Début d'entraînement......... $4 . \bar{c}=\eta_{i} \hat{\alpha}$
Echelle de temps de charriage. $\theta=\eta_{\mathrm{H}} \xi^{-1 / 2}$

\section{C) Conclusions :}

Les deux formules donnent des résultats idenliques en ce qui concerne la vilesse de début d'entrainement et la condition de similitude qui en résulle, $-\mu \xi=n_{i}$, - permet de choisir le diamètre du matériau d'essai lorsque l'on s'est fixé la densité, ou inversement, les échelles géométriques du modèle étant fixées.

En ce qui concerne le charriage établi, les deux formules ne donnent une relation de similitude commune qu'en labsence de distorsion : $\xi=1, \theta=\eta \theta_{\mathrm{II}}$. Cette relation donne l'échelle des lemps de charriage lorsque les caractéristiques du modèle et du matériau d'essai sont définies.

Par contre, pour un modèle distordu, les deux formules conduisent à des relations de similitude différentes :

$$
0=\eta \theta_{\mathrm{H}} \xi^{-3 / 2} \quad \text { oul } \quad \theta=\eta \theta_{\mathrm{H}} \xi^{-1 / 2}
$$

sans que l'expérience des modèles fluviaux distordus à fonds mobiles permette actuellement de donner la préférence à l'une ou l'autre des formules.

\section{II. - LOIS DE CHARRIAGE SOUS L'ACTION DE LA HOULE}

En plus des notations définies plus haut, nous utiliserons les suivantes :

H : amplitude ou creux de la houle;

T : période;

$\mathrm{L}$ : longueur d'onde;

$e^{\prime}$ : masse spécifique apparente (sous l'eau) du matériau de fond;

$U$ : vitese orbitale maximum près du fond, mais en dehors de la couche limite;

$\alpha$ : obliquité de la houle au large par rapport au rivage;

G : débit solide du transport littoral.

\section{A) Etude des conditions de début d'entraînement :}

Les études effectuées au Laboratoire National d'Hydraulique, el dont il a été rendu compte dans une autre communication, amènent à considérer deux cas :

1. Matériaux de grandes dimensions par rapport à l'épaisseur de la couche limite $\mathrm{D}>2,6 \sqrt{v^{\prime} \mathrm{T}}$. C'est le cas des galets.

La condition de début d'entraînement s'écrit : $\mathrm{U}^{2} / \omega_{s}^{\prime} \mathrm{D}=\mathrm{C}^{\mathrm{te}}$, de l'ordre de 3

2. Matériaux de dimensions inférieures à l'épaisseur de la couche limite, I $<2,6 \sqrt{\times^{\top} \mathrm{T}}$. C'est le cas du sable.

La condition de début d'entraînement s'ćcrit :

$$
\mathrm{U}=0,33\left(g / \rho_{0}\right)^{2 / 3} y^{1 / 24} p^{2 / 3} \mathrm{D}^{1 / 4} \mathrm{~T}^{3 / 8}
$$

\section{B) Evaluation du débit solide :}

Des études effectuées dans différents laboraloires et l'interprétation des phénomènes naturels ont conduit à admettre, pour l'évaluation du débit du transport littoral sous l'action d'une houle oblique, une formule du type :

$$
\mathrm{G}=\mathrm{KH}^{2} \mathrm{~T} f(\alpha)
$$

\section{C) Conditions de similitude :}

Nous sommes amenés à distinguer les différents cas suivants :

-...- Modcle sans distorsion;

-.- Modcle avec distorsion, comportant par ailleurs une distorsion des périodes telle que les longueurs d'onde de la houle soient à l'échelle des hauteurs et non à l'échelle des dimensions horizontales $\lambda$. Cette condition est impérative pour que soient respectées les répartitions des vitesses orbitales el les conditions de déferlement;

- Modèle avec ou sans distorsion géométrique, comportant une distorsion des amplitudes de houle qui se traduit par une échelle des amplitudes égale, non à $u$, mais à $\mu . K, K$ pou- 
vant être inférieur ou supérieur à 1 , mais assez voisin de 1, pour que les cambrures, et par conséquent les conditions de déferlement, soient peu affectées.

L'application des formules (4), (5) et (6) à ces différents cas, pour le prototype et le modèle, fournit différentes conditions de similitude que nous examinerons en détail en étudiant leur compatibilité avec celles relatives au charriage par les courants.

\section{I. - CHARRIAGE SIMULTANE SOUS L'ACTON DE LA HOULE ET DES COURANTS - COMPATIBILITE DES CONDITIONS DE SIMILITUDE}

Pour l'étude des domaines maritimes où interviennent simultanément la houle et les courants, nous admettrons que, dès lors que l'une des conditions de mise en mouvement des sédiments en un point donné est atteinte, que ce soit en application de l'une des formules (2), (4) ou (5), le transport des sédiments résulte de l'action simultanée des courants et de la houle, en accord avec les formules (1) ou (3) et (6).

Il en résulte que pour reproduire convenablement les phénomènes naturels sur modèle, il faut et il suffit que les conditions de similitude déduites de ces diverses formules soient compatibles.

C'est ce que nous allons examiner maintenant en détail dans les différents cas envisagés.

Nous avons groupé, en deux tableaux récapitulatif's, les différentes conditions de similitude établies plus haut ainsi que les relations entre les échelles qui permettent de les rendre compatibles.

TABLEA

Matériaux de dimensions relativement grandes par rapport

à l'épaisseur de la couche limite (galets)

\begin{tabular}{|c|c|c|c|c|}
\hline MODE்LE & & DÉBUT D'ENTRAINEMEN'T & $\begin{array}{c}\text { ÉcHELLE } \\
\text { des temps de charriage }\end{array}$ & CONSEQUENCES \\
\hline \multirow{3}{*}{ Sans distorsion. . } & Courant & $\mu=\eta^{\delta}$ & $0=\eta_{11} 0_{11}$ & \multirow{3}{*}{$\begin{array}{l}\text { Similitude possible } \\
\text { pour une infinité } \\
\text { de matériaux. }\end{array}$} \\
\hline & Houle & $\mu=\eta \delta$ & $0=\eta_{1} \theta_{I I}$ & \\
\hline & $\begin{array}{l}\text { Compatibilité } \\
\text { Courant - Houle }\end{array}$ & \multicolumn{2}{|c|}{ Toujours compatibles } & \\
\hline \multirow{3}{*}{ Avec distorsion. . } & Courant & $\mu \xi=\eta^{\hat{o}}$ & $\begin{array}{l}0=\eta \theta_{11} \xi-1 / 2 \\
\text { ou } \eta_{11} \theta^{-3 / 2}\end{array}$ & \multirow{3}{*}{$\begin{array}{l}\text { Similitude } \\
\text { impossible. }\end{array}$} \\
\hline & Houle & $\mu=n \delta$ & $\theta=\eta_{1} \theta_{11} \xi-1$ & \\
\hline & $\begin{array}{c}\text { Compatibilité } \\
\text { Courant - Houle }\end{array}$ & Incompatibilité & $\begin{array}{c}\text { Bonne } \\
\text { compatibilité }\end{array}$ & \\
\hline
\end{tabular}


T A B L E A U I I

Matériaux de dimensions relativement petites par rapport à l'épaisseur de la couche limite (sables)

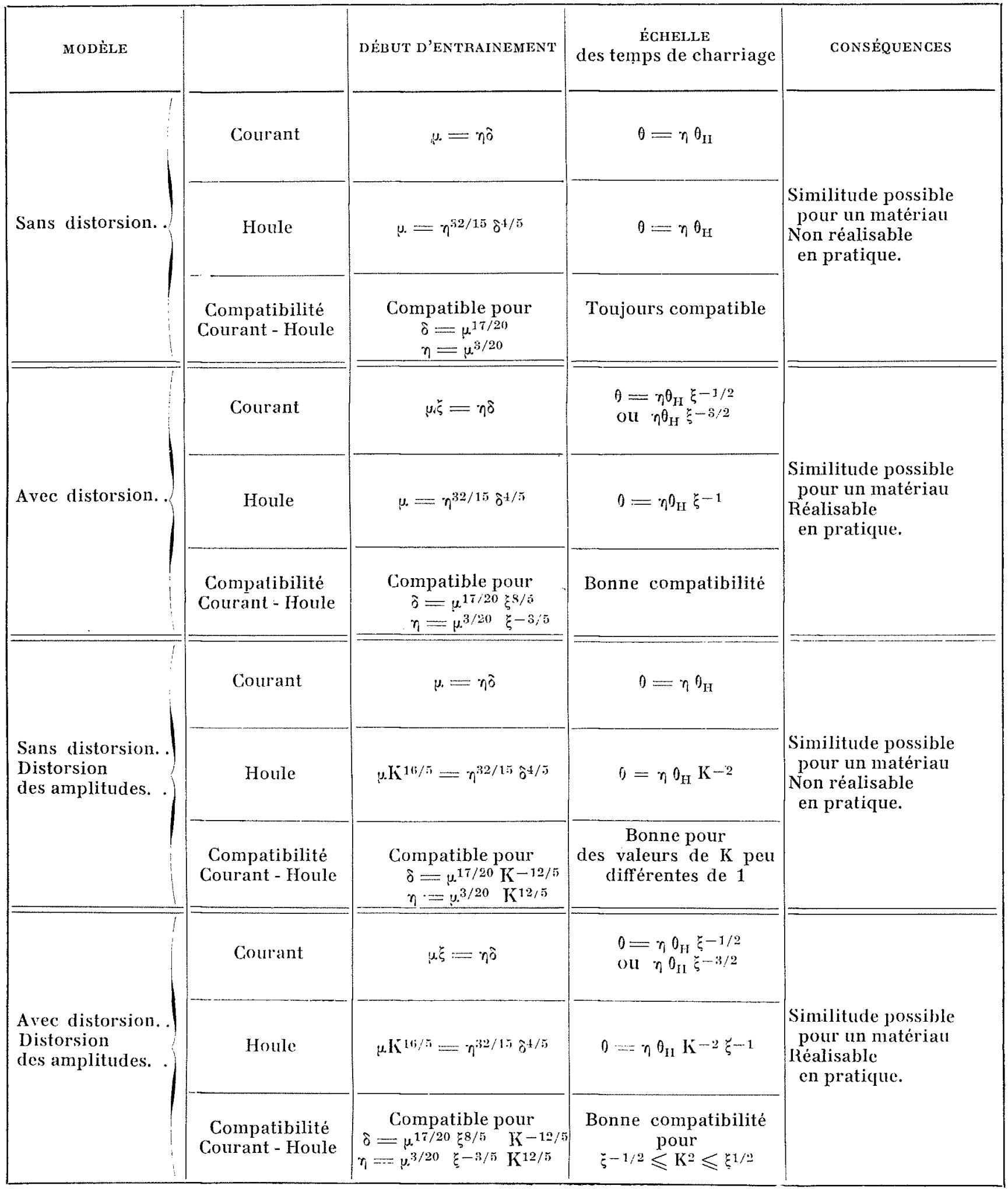


L'examen des tableaux ci-dessus permet de tirer les conclusions suivantes:

\section{A) Transport des sédiments de dimensions relativement grandes par rapport à l'épaisseur de la couche limite (galets).}

\section{MOdÉle SANS DISTORSION :}

La similitude du charriage sous l'action de la houle et des courants est possible pour une infinité de matériaux.

\section{Modkile Distorde :}

On peut considérer que les échelles des temps de charriage sont compatibles puisque l'exposant de $\xi$ dans l'expression du temps de charriage par la houle est intermédiaire entre ceux de ce même paramètre dans les relations déduites des formules de Meyer-Peter et d'Eguiasaroff.

Par contre, les conditions de similitude de début d'entraînement sont incompatibles.

\section{B) Transport des sédiments de dimensions relativement petites par rapport à l'épaisseur de la couche limite (sables).}

\section{MODĖLE SANS DISTORSION :}

Les échelles de temps de charriage sont toujour's compatibles.

En ce qui concerne le début d'entraînement, il est toujours possible de déterminer les caractéristiques d'un matériau respectant à la fois les conditions de similitude établies pour la houle et les courants, mais des difficultés d'ordre pialique font qu'il n'est pas toujours possible d'utiliser le matériau ainsi défini.

On peut remarquer en effet que la compatibilité des conditions de similitude de début d'entraînement, pour un modèle non distordu, implique le respect de la relation $\delta=u^{17 / 20}$ qui impose de choisir un matériau de dimensions sensiblement à l'échelle géométrique $\mu$. Or, par suite de la nécessité d'utiliser des matériaux de granulonétrie assez voisine de celle du sédiment nalurel, pour éviter d'être gêné par les phénomènes de cohésion qui deviennent prépondérants dans les matériaux de granulométrie fine, la relation $\delta=y^{17 / 20}$ est pratiquement impossible à réáliser.

\section{Modelle Distordu :}

Comme dans le cas du transport des galets, les échelles des temps de charriage sont compatibles.
Pour le début d'entrainement, les relations de compatibilité sont les suivantes:

$$
\begin{aligned}
& \delta=\mu^{17 / 20} \xi^{8 / 5} \\
& \eta=\mu^{3 / 20} \xi^{-3 / 5}
\end{aligned}
$$

Nous avons présenté sur un même graphique ces deux relations, pour différentes valeurs de la distorsion $\xi$ (voir fig. 1).

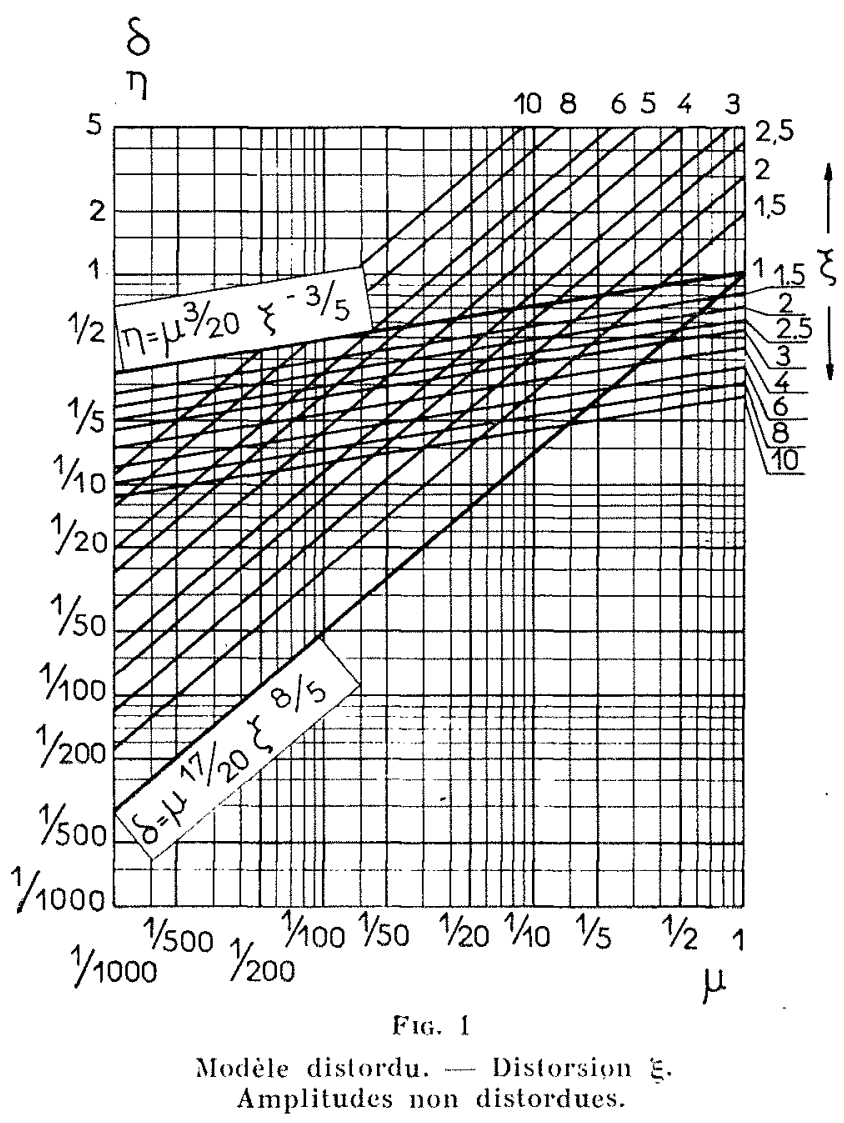

On peut y voir que dans la gamme des échelles couramment utilisées (du $1 / 200^{\circ}$ au $1 / 50^{\circ}$ ), il faut adopter, pour obtenir des valeurs convenables de l'échelle des diamètres $(\geqslant 1 / 5)$, une distorsion géométrique de l'ordre de 4 , qui est encore acceptable.

\section{ModélJe sans Distorsion.-- Amplitudes Dis- TORnUEs :}

Les échelles des temps de charriage sont incompatibles.

Pour une distorsion $K=0,7,1$ 'échelle des temps de charriage par la houle est déjà multipliée par 2, alors que, comme le montre le graphique de la figure 2, il faut adopter une distorsion $\leqslant$ à 0,5 pour obtenir une échelle des diamètres de l'ordre de $1 / 5$. 


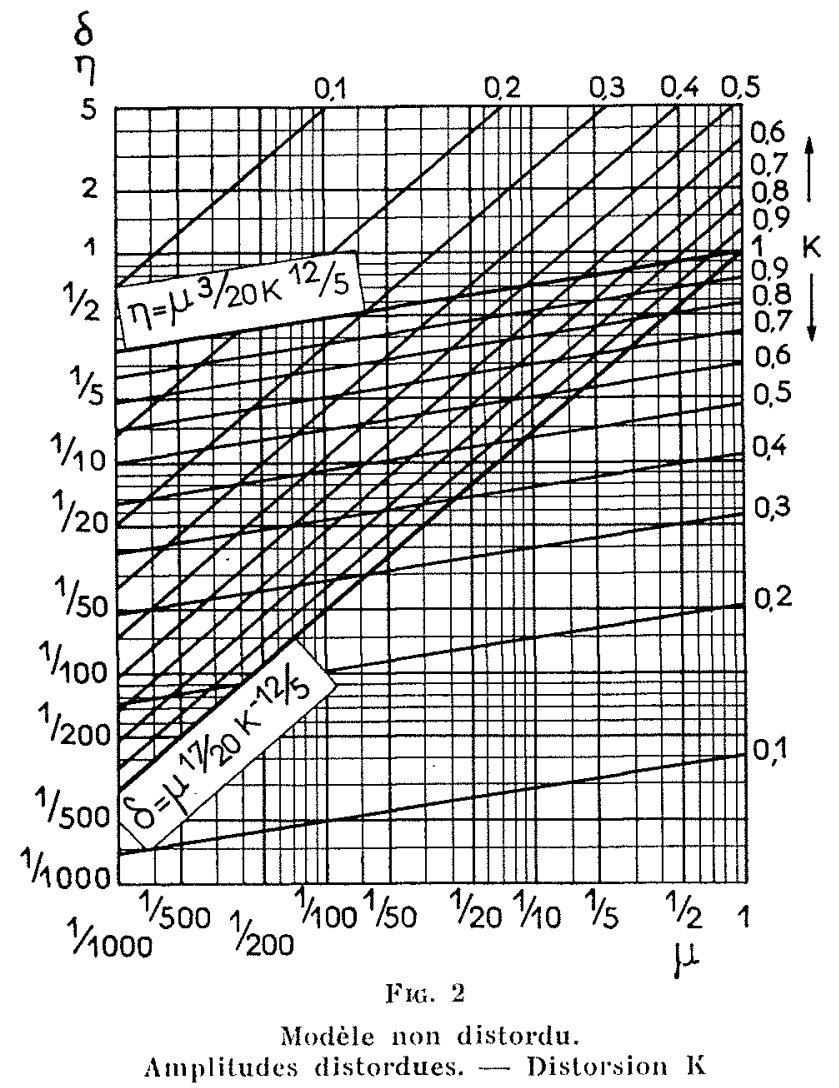

\section{Modéle pistordu. - Amplitudes} DISTORDUES :

Les échelles des lemps de charriage sont les suivantes :

$$
\begin{array}{ll}
\text { Courant. . } & 0=\eta \theta_{\mathrm{H}} \xi^{-1 / 2} \text { ou } 0=r_{1} \xi^{-3 / 2} \\
\text { Houle. . . } & 0=\eta \theta_{\mathrm{H}} \xi^{-1} \mathrm{~K}-2
\end{array}
$$

Elles restent compatibles pour $\xi^{-1 / 2} \leqslant \mathrm{~K}^{2} \leqslant \xi^{1 / 2}$, soit, à la limite inférieure $\xi^{-1 / 2}=\mathrm{K}^{2}$, pour $K=\xi^{-1 / 4}$.

Pour le début d'entraînement, les relations de compatibilité :

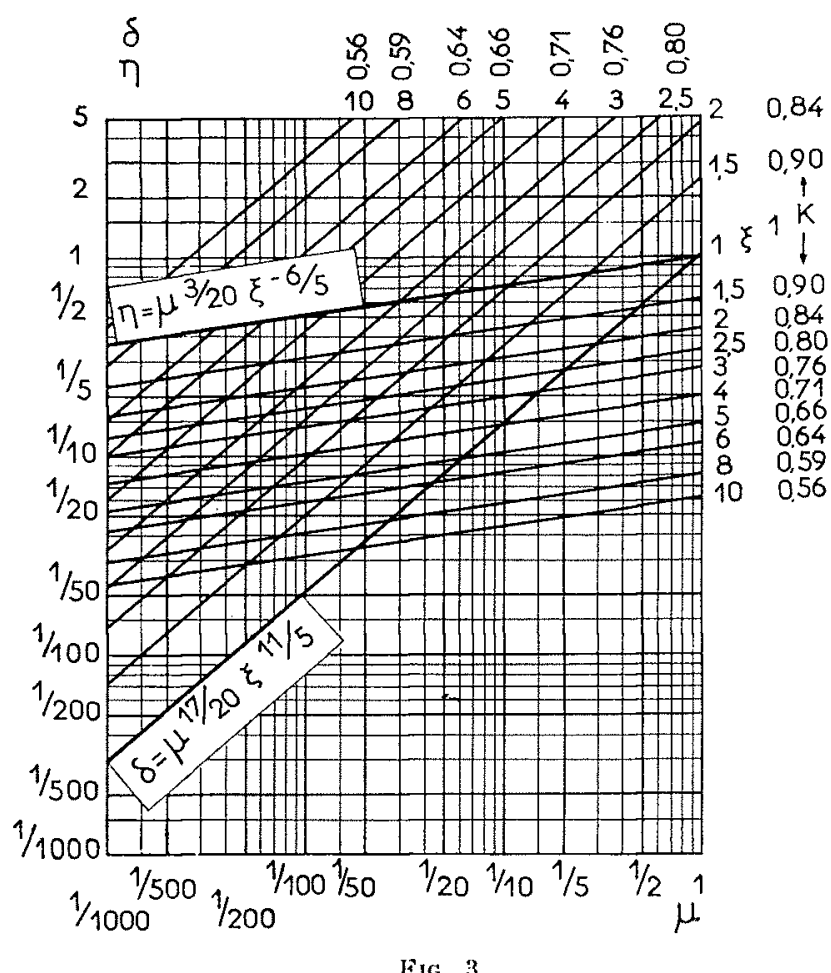

Modèle distordu. - Distorsion $\varepsilon$. Amplitudes distordues, - Distorsion $\mathrm{k}=\xi^{-1 / 4}$.

$$
\begin{aligned}
& \varepsilon=\mu^{17 / 20} \xi^{8 / 5} \quad \mathrm{~K}^{-12 / 5} \\
& \eta=\mu^{3 / 20} \xi^{-3 / 5} \mathrm{~K}^{12 / 5}
\end{aligned}
$$

deviennent alors:

$$
\begin{aligned}
& \delta=\mu^{17 / 20} \xi^{11 / \hbar} \\
& \eta=\mu^{3 / 20} \xi-6 / 5
\end{aligned}
$$

Ces deux relations sont représentées graphiquement sur la figure 3 . On peut $y$ voir, en se reportant par ailleurs à la figure 1 , que la combinaison des deux distorsions offre une plus grande souplesse dans le choix des caractéristiques du modèle et du matériau, en permettant, à même échelle des diamètres, de diminuer la distorsion ou inversement pour une même valeur de la distorsion, d'augmenter l'échelle des diamètres.

\section{IV. - EXEMPLES D'APPLICATIONS}

Comme nous l'avons déjà dit plus haut, un certain nombre d'études mettant en jeu l'action simultanée de la houle et des courants sur les sédiments ont été effectuées à Chatou, antérieurement à la présente mise au point.
Nous allons voir dans quelle mesure les conclusions des essais d'étalonnage de ces études vérifient les conditions de similitude que nous venons de proposer. 


\section{A) Etude de l'aéroport de Nice :}

L'ćtude de l'extension par emprise en mer de l'aéroport de Nice fait actuellement l'objet d'essais sur un modèle réduit à l'échelle du $1 / 200^{\circ}$ sans distorsion.

Le but de cette étude est de vérifier si la modification du tracé de la ligne de rivage actuelle, entrainée par les travaux d'extension, ne risquait pas de rompre l'équilibre de la côte et de provoquer, à plus ou moins longue échéance, des érosions du littoral, de part et d'autre de l'aéroport. (Voir fig. 4.) struction du port de Cros-de-Cagnes par les galets sous l'action des houles de direction $\mathrm{SE}$ à S-SE.

Nous nous trouvions pour ce problème dans le cas de matériaux de grandes dimensions vis-à-vis de l'épaisseur de la couche limite, sur un modèle non distordu et pour lesquels, par conséquent, les conditions de similitude de charriage sous l'action de la houle et des courants sont toujours compatibles.

Les galets du Var et du littoral ont étẻ représentés par du pollopas, de densité 1,39 et de granulométrie comprise entre 0,04 et $2 \mathrm{~mm}$, dont

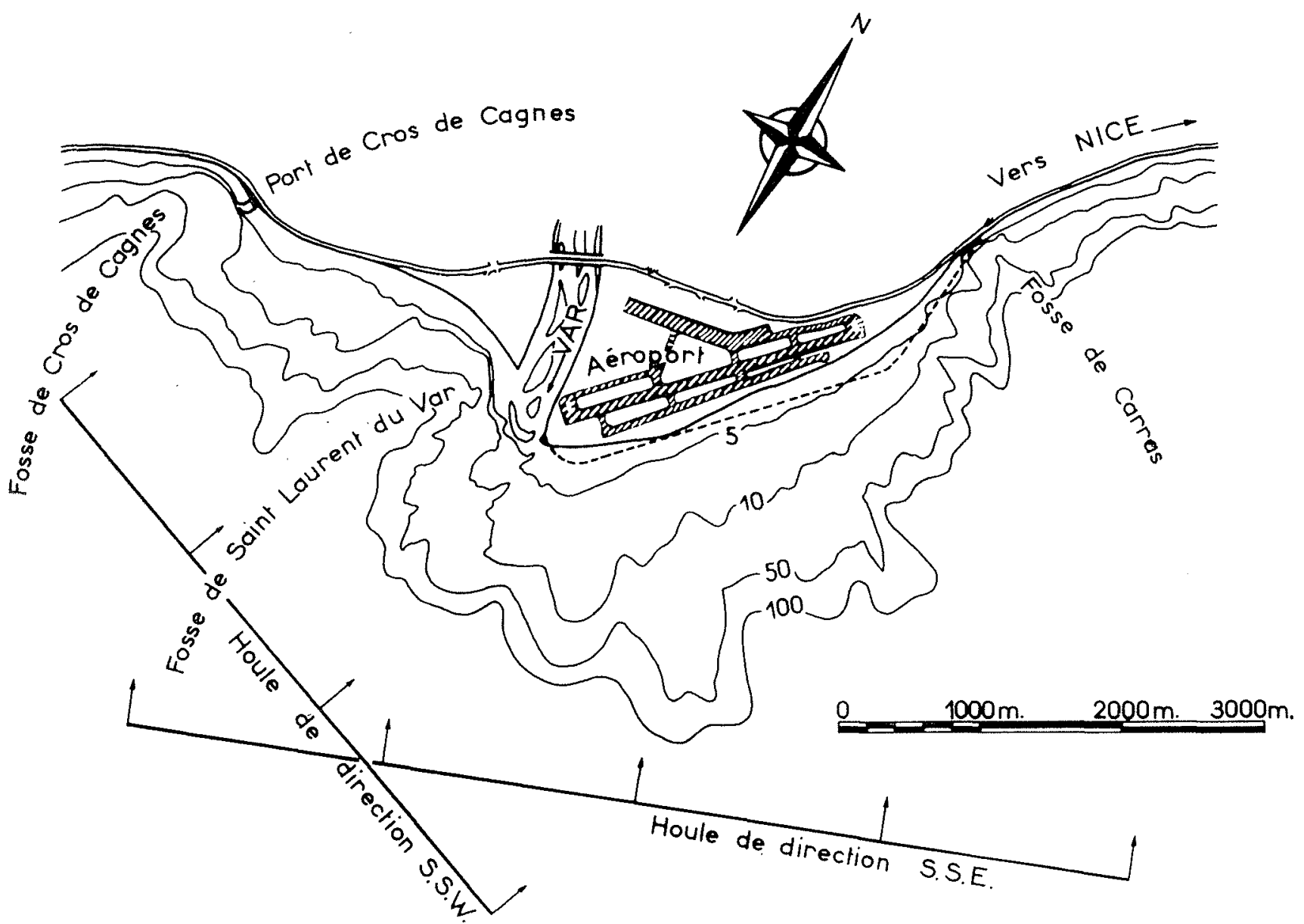

Fig. 4

Carle du liltoral at voisinage de laéroporl de Nice.

Elle a comporté, comme toules les études de ce genre, une phase d'essais d'étalonnage au cours de laquelle nous avons cherché à représenter les phénomènes naturels. Nous nous sommes attachés, en particulier, à reproduire l'apport des galets à la mer par les crues du Var et leur répartition le long du littoral, de part et d'autre de l'embouchure, sous la forme de cordons littoraux, ainsi que le phénomène d'ob- les échelles :

$$
\eta=1 / 4,4 \quad \text { el } \quad \delta=1 / 35 \text { à } 1 / 50
$$

vérifient bien la condition de début d'entrainement :

$$
\mu=n^{\delta}=1 / 200
$$

La relation $\theta=n 0_{\mathrm{II}}$ donne par ailleurs la valeur de l'échelle des temps de charriage $\theta=1 / 60$. 


\section{VÉRIFICATIONS EXPÉRIMENTALES}

\section{Débu't D'Entraînement :}

Au cours d'une campagne de mesures sur place effectuée par le Service des Ponts et Chaussées des Alpes-Maritimes, on a pu déterminer, à l'aide de traceurs colorés et de traceurs radioactifs, les conditions de début d'entraînement des galets dans le Var.

Le début de charriage se produit pour des débits de l'ordre de $180 \mathrm{~m}^{3} / \mathrm{s}$. Il a été reproduit sur le modèle dans des conditions de débits homologues.

\section{EChelle des 'TEMPS DE CIIARRIAGE:}

Les conditions d'obstruction de la passe d'entrée du port de Cros-de-Cagnes par les galets en provenance de l'est ont pu être observées avec suffisamment de précision pour nous permettre de vérifier la valeur théorique de l'échelle des temps de charriage.

L'obstruction du port, observée après une période de trois jours de tempête de direction S-SE, a été reproduite sur le modèle, pour des conditions de houle homologue dans un temps compris entre 60 et 70 minutes.

L'équivalence entre les durées des phénomènes observés en nature et sur modèle donne à l'échẹlle des temps de charriage la valeur expérimentale $\theta=1 / 60$ à $1 / 70$.

Par ailleurs, nous avons cherché, en utilisant un piège à galets, à déterminer le débit solide pour en déduire une valeur de l'échelle des temps de charriage, mais, devant les difficultés rencontrées, nous avons renoncé à ces mesures, pour comparer les vitesses de déplacement des galets, dans la nature et sur le modèle, qui sont relativement faciles à mesurer.

En faisant l'hypothèse que le déplacement des galets se fait par le mouvement continu de $n$ couches de galets de diamètre moyen $d_{m}$, dans le modèle comme en nature, sur une longueur $l$ perpendiculairement au rivage, et compte tenu des relations $\mu=\eta_{\grave{\delta}}$ et $0=r_{i} \theta_{n}$, l'échelle des vitesses de transfert est donnée par :

$$
\lambda_{\mathrm{v} t}=\mu^{1 / 2}=0_{\mathrm{I} 1}
$$

Les premières comparaisons des mesures de vitesses effectuées en nature et sur le modèle, en des points et dans des conditions de houle homologues, semblent bien confirmer la relation $\lambda_{\mathrm{y} t}=\theta_{\mathrm{H}}$ et en conséquence, la validité de la relation $\theta=\eta \theta_{\mathrm{H}}$, qui donne à $\theta$ la valeur $1 / 60$.

\section{B) Etude de la digue du port de Cotonou :}

A l'occasion d'une étude des affouillements au pied d'une digue destinée au port de Cotonou, nous avons été amenés à rechercher un matériau respectant à la fois les conditions de mise en mouvement et de charriage sous l'action simultanée de la houle et des courants.

Compte tenu des délais extrêmement courts qui nous étaient impartis pour l'exécution de cette étude, nous avons jugé préférable d'utiliser des matériaux en stock au Laboratoire et de déterminer l'échelle géométrique du modèle qui permettrait de respecter simultanément les conditions de début d'entrainement sous l'action de la houle et des courants.

Il s'agissait de représenter, sur un modèle sans distorsion, à une échelle comprise entre le $1 / 60^{\circ}$ et le $\mathbf{1} / 15^{\mathrm{e}}$, les mouvements d'un sable de densité 2,6 et de $0,5 \mathrm{~mm}$ de diamètre moyen. Nous disposions en stock des matériaux suivants :

-- du lignite de densité 1,36 et de diamètre moyen $0,6 \mathrm{~mm}$

-- du plexiglas de densité 1,18 et de diamètre moyen $0,45 \mathrm{~mm}$

- du polystyrène de densité 1,06 et de diamètre moyen $0,6 \mathrm{~mm}$

dont les échelles étaient les suivantes :

$$
\begin{array}{lll}
\text { - lignite . . . } & \delta=1,2 & \eta=1 / 4,5 \\
\text { - plexiglas . . } & \delta=1 / 1,1 & \eta=1 / 9 \\
\text { - polystyrène . . } & \delta=1,2 & \eta=1 / 27
\end{array}
$$

Comme il fallait s'y altendre, aucun de ces matériaux ne permet de respecter de façon rigoureuse la similitude du début d'entrainement simultané sous l'action de la houle et des courants, qui, comme nous l'avons vu, impose les relations de compatibilité :

$$
\delta=\mu^{1 \pi / 20}, \quad \eta=\mu^{3 / 20}
$$

Ces relations donnent, pour un modèle sans distorsion, à une échelle comprise entre le $1 / 50^{\circ}$ et le $1 / 20^{\circ}$, un matériau mobile dont les échelles sont comprises entre le $1 / 28^{\circ}$ et le $1 / 13$ pour les diamètres et entre le $1 / 1,8$ et le $1 / 1,3$ pour les densités. L'échelle géométrique a été déterminée graphiquement de la façon suivante:

- En portant sur un graphique (fig. 5), en fonction de l'échelle géométrique $\mu$, et respectivement pour la houle et les courants, les vitesses correspondant au début d'entraînement, d'une part pour les matériaux dont nous dispo- 


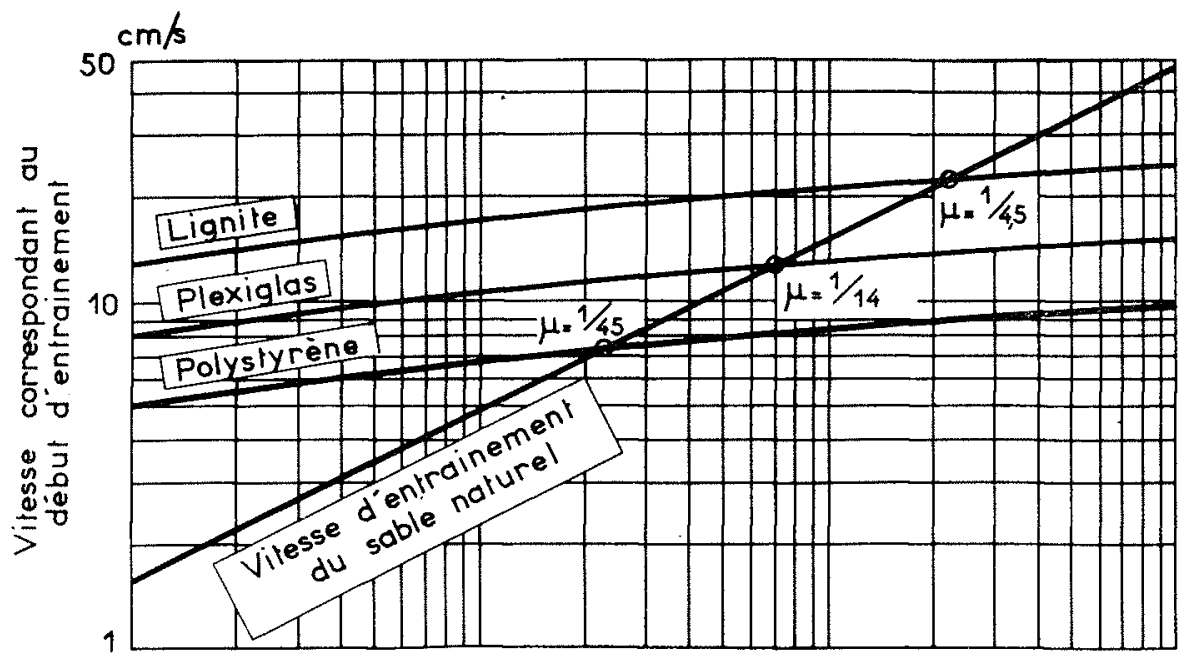

Entrainement por

les couranis

(Durand el Condolios)

$\frac{V}{\sqrt{g d \cdot \frac{\sqrt{P \cdot P_{0}}}{P_{0}}}}=A \log \frac{h}{D}+B$

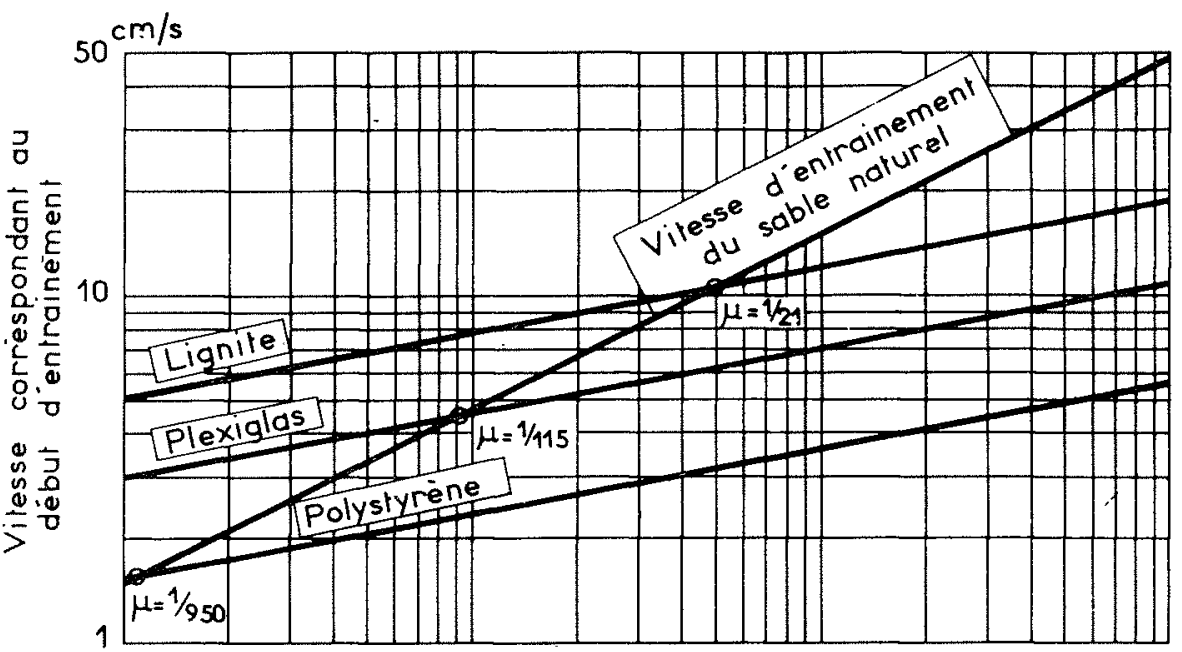

Entrainement par la houle

$V=0,33\left(\frac{g}{P_{0}}\right)^{2 / 3} \nu^{1 / 24} \rho^{2 / 3} D^{1 / 4} T^{3 / 8}$

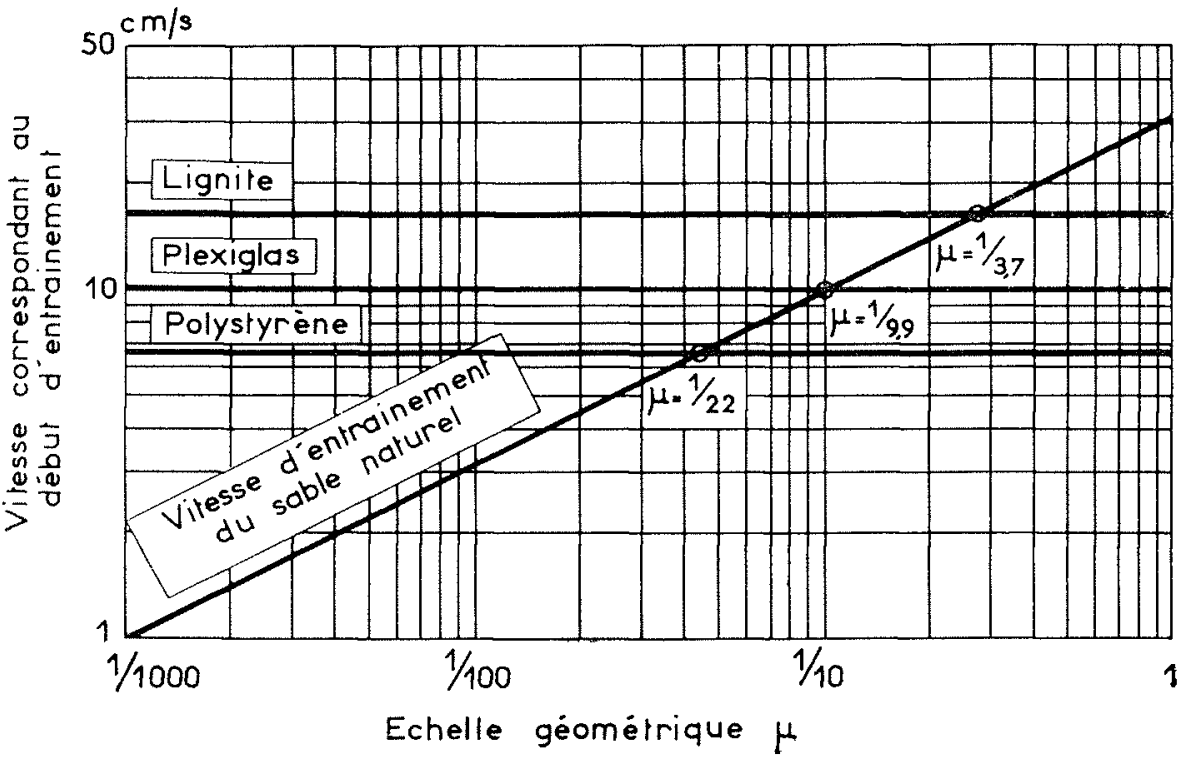

Entrainement par

les courants (Meyer. Peler)

$$
\begin{gathered}
U=\sqrt{\lambda / 8} \cdot \sqrt{\tau_{0} / P_{0}} \\
\tau_{0}=A \omega_{S}^{\prime} D
\end{gathered}
$$




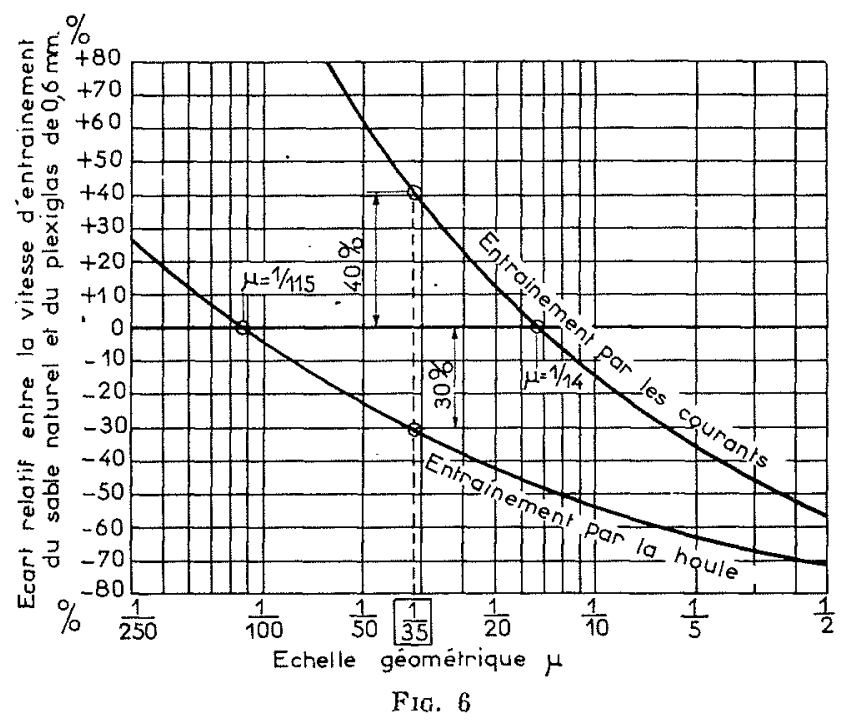

sions, d'autre part pour le sable naturel, nous obtenons les valeur's de l'échelle à adopter pour respecter la condition de début d'entrainement, soit par la houle, soit par les courants.

Nous avons finalement choisi le plexiglas et l'échelle du $1 / 35^{\circ}$, intermédiaire entre le $1 / 115^{\circ}$, correspondant à l'entraînement par la houle et le $1 / 14^{\circ}$ correspondant à l'entraînement par les courants.

Le graphique de la figure 6, sur lequel nous avons porté, en fonction de l'échelle géométrique, les écarts relatifs entre les vitesses d'entrainement pour le sable naturel et pour le plexiglas, montre que pour l'échelle choisie, le plexiglas présente une mobilité trop grande $(30 \%)$ sous l'action de la houle, et au contraire, dans une proportion du même ordre $(40 \%)$, une mobilité trop faible sous l'action des courants.

\section{CONCLUSIONS}

Comme nous l'avons déjà souligné dans notre introduction, la présente étude n'a pas l'ambition de résoudre définitivement le problème de similitude du charriage simultané sous l'action de la houle et des courants.

Notre objectif était, dans l'état actuel de nos connaissances, de tenter de nous dégager de l'empirisme qui préside encore au choix des caractéristiques des modèles réduits et des matériaux d'essais, lor'squ'il s'agit de problèmes faisant intervenir à la fois la houle el les courants permanents ou lentement variables.

Nous avons été amenés aux conclusions suivantes, qui ne sont évidemment valables que dans les limites de validité des lois que nous avons utilisées - et nous sarons que ces dernières, établies dans des domaines plus ou moins étendus, sont encore loin de présenter un caractère de généralité.

\section{a) Pour le charri.uge dfs galets :}

- Les conditions de similitude de charriage sous l'action de la houle et des cou- rants ne sont compatibles que dans un modèle sans distorsion.

- Il existe théoriquement dans ce cas une infinité de matériaux répondant aux conditions de similitude.

\section{b) Poun te Charriage des sabi.es:}

- Les conditions de similitude de charriage sont toujours compatibles pour un matériau déterminé, mais, pour un modèle sans distorsion, elles conduisent à choisir des matériaux de granulométrie trop fine pour être pratiquement utilisables.

- Pour rester dans la gamme des matériaux de granulométrie convenable, il faut accepter le principe de la distorsion, la meilleure solution consistant à adopter une distorsion géométrique $\xi$ combinée à une distorsion des amplitudes de houle $\mathrm{K}$, telle que :

$$
\mathrm{K}=\xi^{-1 / 4}
$$

\section{I S C U S S I O N}

président: M. Knavtchenko

M. le Président remercie vivement M. JafFry de son très intéressant exposé.

M. le Président demande ensuite s'il y a d'autres raisons que des raisons expérimentales de croire que les effets de la houle et ceux des courants se superposent linéairement.

M. JAFFry ayant répondu négativement, en précisant qu'il n'est même pas certain que les phẻnomènes soient additifs, M. le Président exprime le veeu que cette hypothèse de base soit très clairement indiquée dans le mémoire dont le très grand intèrêt est, précisément, d'ouvrir la voie à une vérification expérimentale de cette loi linéaire.

M. Godder complète l'exposé de M. JAFFry par les remarques suivantes: 
« I.étude de la similitude des sédiments sous l'action simultanée de la houle et des courants, envisagée dans la présente communication, a pour but de déterminer un matériau mobile susceptible de reproduire les mouvements de sédiments sur un modèle réduit où interviennent à la fois la houle et les courants, en admettant que l'un des deux facteurs est prépondérant en un point donné de la zone maritime étudiée. G'est le cas du modèle de l'áéroport de Nice cité en exemple.

"Pour prouver que les conditions de similitude indiquées sont suffisantes à la reproduction des mouvements de fonds en un point où les deux facteurs ci-dessus interviendraient simultanément avec une importance comparable, il faudrait montrer que les lois d'entraîne- ment sont additives en ce qui concerne les vitesses des particules d'eau résultant, d'une part de la houle, d'autre part des courants. »

M. JAFFry confirme l'interprétation de $M$. le Président en ce qui concerne le résultat des mesures, à savoir qu'il plaide en faveur des vérifications expérimentales, encore un peu réduites.

M. le Président précise qu'au Laboratoire de Mécanique des Fluides de Grenoble, il a toujours été envisagé d'inscrire au programme de recherches des questions de cette nature. M. le Président pense que les futurs spécialistes trouveront dans l'exposé de MM. Godner et JaFFry, qu'il tient encore à féliciter, un point de départ de leurs futures recherches qu'il souhaite aussi fécondes.

\section{ERRATA}

concernant l'article de M. R. Cartien : "Aménagement hydroélectrique de Roselend (Savoie). Conduite forcée de l'usine de La Bâthie (The Roselend hydroelectric development. The La Bathie power house penstock) 》, paru dans notre numéro 1 de 1960.

\section{Page $13,2^{\circ}$ colonne :}

ligne 19, au lieu de $\Delta \sigma_{2}=\frac{\sigma_{1} \varepsilon}{e^{\prime}}$, lire : $\Delta \sigma_{2}=\frac{\sigma_{2} \varepsilon}{e^{\prime}}$

ligne 22, au lieu de $\Delta \sigma_{b}=\frac{\sigma_{1} \varepsilon}{e^{\prime}}$, lire : $\Delta \sigma_{b}=-\frac{\sigma_{y} \varepsilon}{e^{\prime}}$

Page 15, $1^{\text {re }}$ colonne :

ligne 19, an lien de $\Delta \sigma_{1}= \pm \frac{\sigma_{1} \varepsilon}{e^{\prime}}$, lire: $\Delta \sigma_{2}= \pm \frac{\sigma_{. . \varepsilon}}{e^{\prime}}$

ligne 22, au lieu de $\sigma_{3}=\sigma_{2}+\left(\Delta \sigma_{1}\right)$, lire : $\sigma_{3}=\sigma_{2}+\left(\Delta \sigma_{2}\right)$

ligne 23, au lieu de $\sigma_{c}=\sigma_{h}-\left(\Delta \sigma_{1}\right)$, lire : $\sigma_{c}=\sigma_{b}--\left(\Delta \sigma_{2}\right)$ 